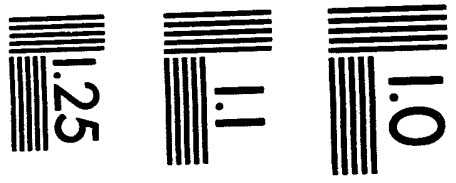

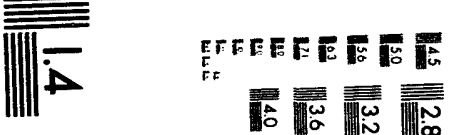

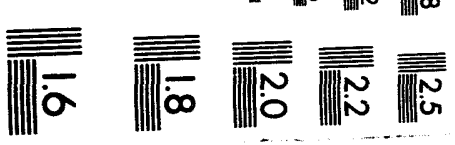



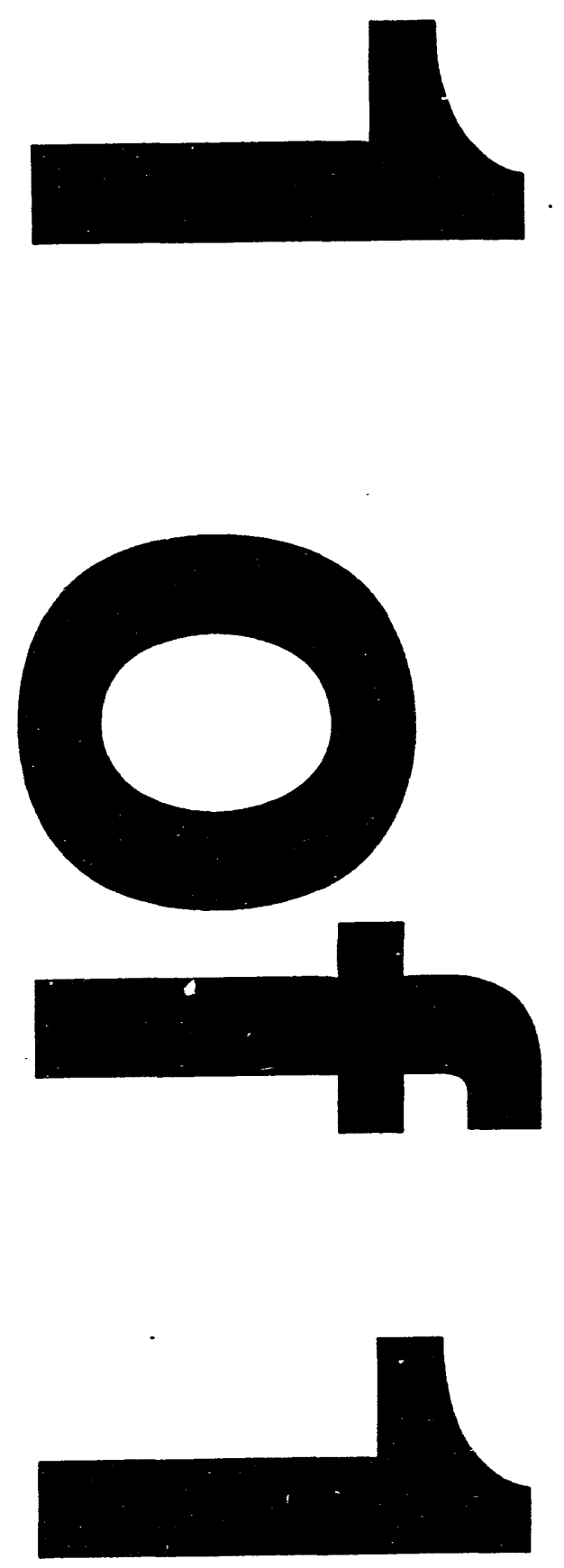


\title{
Guiding-center equations for electrons in ultraintense laser fields
}

\author{
Joel E. Moore and Nathaniel J. Fisch
}

Princeton Plasma Physics Laboratory

Princeton University, P. O. Box 451, Princeton, NJ 08543

\begin{abstract}
The guiding-center equations are derived for electrons in arbitrarily intense laser fields also subject to external fields and ponderomotive forces. Exhibiting the relativistic mass increase of the oscillating electrons, a simple frame-invariant equation is shown to govern the behavior of the electrons for sufficiently weak background fields and ponderomotive forces. The parameter regime for which such a formulation is valid is made precise, and some predictions of the equation are checked by numerical simulation.
\end{abstract}

PACS numbers: $\quad 52.40 . \mathrm{N} \quad 52.50 . \mathrm{J} \quad 52.75 . \mathrm{M}$ 


\section{INTRODUCTION}

The increasing degree of interest in high-intensity lasers $\left(a=e E_{0} / m c \omega \sim 1\right)$ motivates a theoretical examination of the behavior of electrons oscillating in the fields of such lasers. The electron motion is well understood when the only forces present are those from the wave ${ }^{1}$; this paper examines the motion of electrons when other fields are present in addition to the wave.

The nonlinearity parameter $a$ can be understood as the ratio of the momentum imparted by the wave field in a single oscillation to mc. (For $\lambda=1 \mu \mathrm{m}, a=0.84$ at an intensity of $I=c E_{0}{ }^{2} / 8 \pi=10^{18} \mathrm{~W} / \mathrm{cm}^{2}$.) As $a \sim 1$, the quiver velocity of an electron in the wave becomes relativistic, and, as a result, the magnetic component of the wave begins to affect the motion. Because the electron velocity is relativistic over much of the orbit, the electrons can exhibit an increased "effective mass" or increased inertia to applied forces. While effective mass equations do appear in the literature, there remains a need for a general and systematic derivation of such equations, including precise statements concerning the limitations of such approximations. We show here that the non-oscillatory part of the electron motion is given by a simple guiding-center equation, which predicts some interesting results, verified here by numerical simulation.

The formulation developed here is useful in many situations of practical importance where electromagnetic fields in addition to the wave field are present, and where these background fields are much weaker than the wave field and vary only slightly over oscillation time and space scales. For example, longitudinal electron oscillations in a plasma occur with characteristic time $1 / \omega_{p} \gg 1 / \omega$ and space scale roughly the Debye length $\lambda_{D}$, which may be larger or smaller than the beam wavelength. Plasma oscillations in the presence of laser pulses play key roles in the beat wave accelerator $^{2}$ and wakefield accelerator. ${ }^{3}$ Close-encounter collisions involving oscillating electrons can occur on time and space scales smaller than those of the oscillations, and in this case the enhanced-mass picture ceases to be valid, but a different form of the equations derived here can be applied to this case as well.

Various accelerator schemes ${ }^{4-6}$ attempt some sort of conversion of the intense transverse fields of a laser into a more useful form, and constraints on when such conversion can occur are given here. The general constraints given here confirm and generalize the constraint found by Apollonov 
et al..$^{6}$ for a specific accelerator design, and also explain the optimal parameters Kawata et al. found numerically for two accelerator designs. ${ }^{5-6}$ Essentially these accelerator designs convert some of the relativistic quiver velocity into drift velocity, and this can only be done with certain types of background fields. Powerful laser pulses in the presence of background fields may also have applications as diagnostic tools in plasmas. ${ }^{7}$

Electrons struck by a laser pulse experience a ponderomotive acceleration at the beginning of the pulse that can result in a relativistic drift velocity (distinct from the quiver velocity) during the body of the pulse. ${ }^{8}$ It turns out that the ponderomotive acceleration appears naturally through an analysis of these drift velocities. We derive a simple equation, (40), which governs the timeaveraged behavior of oscillating electrons in the presence of weak background fields and/or weak ponderomotive gradients, for incident plane waves of arbitrary intensity. We show that, in some ways, the character of the ponderomotive force in the presence of additional fields is changed. For example, in the absence of background fields, the ponderomotive force, taken over the whole wave, only displaces the particle and does not change its energy, while, in the presence of a background electric or magnetic field, energy transfer from the ponderomotive force can take place.

It will be assumed throughout that the pulse has no transverse variation and has phase velocity equal to the speed of light in vacuo. The small spot sizes required to achieve very high intensity do create some transverse variation of the pulse, but in many cases this variation is small over a single electron's orbit. For vacuum applications the assumption that the phase speed is equal to $c$ clearly poses no problems, although in plasma applications the phase speed may vary. Often plasmas irradiated by intense lasers are quite underdense, so that the deviation of the phase velocity from $c$ is small. Longitudinal variation of the pulse leads to the well-known ponderomotive effect, which is considered here in some detail. Transverse variation of the pulse leads both to transverse ponderomotive effects and to waveguide-like longitudinal fields; while these effects can be significant and useful, as in ponderomotive focussing ${ }^{9}$ and longitudinal acceleration ${ }^{10}$, in many applications they are either small in magnitude or affect only a small fraction of the irradiated electrons. The analysis is specific to the case of nearly monochromatic laser light, because in the nonlinear regime the superposition of frequency components becomes very complicated ${ }^{11}$. 
Some predictions of the analytical framework derived here are checked with a short computer code that integrates the Lorentz force equations numerically. The code is also used to examine regimes beyond the scope of the equations here, in order to understand qualitatively the chrnges in the electron motion.

In Section II the guiding-center equations are found by using special features of the drift solutions of electromagnetic waves. Section III details the assumptions leading to the guiding-center equations and relates the conditions for its validity to optimal designs for certain accelerators. In Section IV, numerical examples are used to show, when conditions for its validity are not satisfied, the breakdown of the guiding center equations and the subsequent interesting dynamics. Section $\mathrm{V}$ examines the classes of fields that allow the guiding-center equations to be averaged to a simpler form. Section VI derives guiding-center equations that are frame-invariant, including the ponderomotive force in the presence of background fields. Our main results are summarized in Section VII.

\section{DERIVATION OF GUIDING-CENTER EQUATIONS}

To derive the guiding center equations, we treat a background force as a series of closely-spaced impulses. Between the impulses, the electron is subject only to the intense electromagnetic wave. The size of the impulse is approximated as the integrated force over the unperturbed trajectory of the electron in the intense wave in the time interval between the impulses. Then the effect of the background force is updated at each impulse, and such an approximation converges in the limit of infinitesimally spaced impulses. (This approach is similar to the Picard method used in the theory of differential equations ${ }^{12}$ ) Certain features of plane waves make it possible to find the response for all times from an arbitrary impulse; then, if it is possible to integrate over the applied impulses, the electron trajectory can be determined. The only approximations and constraints enter at the integration stage.

This approach leads to tractable eqations for the problem at hand for two reasons: one, exact analytic solutions can be written for electrons in arbitrarily intense electromagnetic plane waves ${ }^{13}$; and, two, plane waves remain plane waves in any frame of reference, so that the exact solutions are 
applicable as the particle drifts. These two properties of intense plane waves can be used to derive a simple and accurate guiding-center equation in the presence of external forces, as we now show.

First, note that there are exact analytical solutions for the motion of individual electrons in a plane, monochromatic wave of any polarization (see e.g. Landau and Lifshitz ${ }^{13}$ for the case of linearly and circularly polarized waves; the case of elliptical polarization can be handled similarly). Integrability is a consequence of the existence of three integrals of the motion. ${ }^{14}$ The canonical momenta

$$
P_{y}=p_{y}+e A_{y} / c, \quad P_{z}=p_{z}+e A_{z} / c
$$

are conserved, because of the system's symme',ry with regard to translations perpendicular to the direction of the wave's propagation. Here, $\mathbf{p}$ denotes the kinematic momentum and $\mathbf{P}$ the canonical momentum, with the direction $\hat{\mathbf{k}}$ of the wave is taken along the $\boldsymbol{x}$-axis. In addition, there is a third invariant of the motion $m c \gamma-p_{z}$ (here $\gamma$ is the Lorentz factor of the electron), associated with the dependence of the problem on $x$ and $t$ only through the phase $\eta=\omega(t-x / c)$. The exact solutions of the motion can be derived from these three constants of the motion. In the presence of background fields, however weak, these three quantities are not necessarily conserved, raising the question of how the motion is modified.

Second, note that a plane wave in one frame appears as a plane wave in all other frames, though possibly with a different direction, frequency, and amplitude. Monochromatic waves remain monochromatic, however, and polarization (i.e., eccentricity of polarization ellipse) is also invariant. The norm of the vector potential, $\sqrt{A_{\mu} A^{\mu}}$, which is just $c E_{0} / \omega$ for a linearly polarized wave at a crest is invariant, so that a strength parameter a can be invariantly defined by

$$
a=\frac{m e \sqrt{2\left\langle A_{\mu} A^{\mu}\right\rangle}}{c^{2}}
$$

with \langle\rangle denoting an average with regard to phase. (Note that some other authors define $a$ without the factor of 2.) Thus, given a drift velocity $\mathbf{v}_{d}$ relative to the lab frame and a plane monochromatic wave in the lab frame, there exists a solution of the equations of motion for which the average value of the electron velocity, as calculated in the lab frame, is $\mathbf{v}_{d}$. In the frame moving with velocity $\mathbf{v}_{d}$ relative to the lab frame, the electron has zero average velocity, and in this frame the electron's path is a circle for circularly polarized light and a figure-eight for linearly polarized light. ${ }^{13}$ 
Actually, in our analysis we will use the inverse theorem: given any instantaneous velocity for the electron $v$, and the phase $\eta$ of the wave at the electron's location, there exists a unique frame moving with some velocity $\mathrm{v}_{d}$, different from $v$, in which the electron is moving in a stationary figure-eight orbit. (Some subtleties do arise in the definition of the drift velocity when background fields are present. The definition to be used here is that the drift velocity is the average velocity that the electron would have if all background fields were instantaneously eliminated. For further discussion, see App. A.) There are explicit functions $p_{\perp}(\eta)$ and $p_{\|}(\eta)$, depending on the wave only through $a$, that specify the components of the electron momentum perpendicular to and parallel to the wave axis in the rest frame $\left(p_{\perp}\right.$ is a single function for linear polarization and two functions for circular polarization; the idea is the same). The functions $p_{\perp}$ and $p_{\|}$are calculated explicity in Landau and Lifshitz. ${ }^{13}$ In the unique rest frame of the electron, the components of the electron momentum parallel to and perpendicular to the wave axis are $p_{\perp}$ and $p_{\|}$.

At time $t_{0}$, suppose that the electron has zero drift velocity in the frame where our coordinates are defined. In this frame define $\hat{\mathbf{x}}$ parallel to $\hat{\mathbf{k}}$, the wave axis. After a small time step $d t$, the electron has position and momentum given by

$$
\mathbf{x}\left(t_{0}+d t\right)=\mathbf{x}\left(t_{0}\right)+\mathbf{v}\left(t_{0}\right) d t+O\left(d t^{2}\right), \quad \mathbf{p}\left(t_{0}+d t\right)=\mathbf{p}\left(t_{0}\right)+\mathbf{F}_{\text {wave }} d t+\mathbf{F}_{\text {ext }} d t+O\left(d t^{2}\right)
$$

Here $\mathbf{F}_{\text {wave }}$ and $\mathbf{F}_{\text {ext }}$ are the forces on the electron from the wave and the background fields. After this time step, the wave has some phase $\eta$ that is unchanged by frame switches (the product of the wave and position four-vectors). Our task is to determine the drift velocity $\mathbf{d v}_{d}$ of the frame in which the electron would be at rest on average if the applied force $F_{\text {ext }}$ went to zero after the time $d t$. We know that $\mathbf{p}\left(t_{0}\right)+\mathbf{F}_{\text {wave }} d t$ satisfies the equations of motion at phase $\eta$ in the original frame, i.e., in the absence of the background fields this value for the momentum would be part of an oscillation with zero average velocity in the original frame. In other words,

$$
\begin{aligned}
& p_{x}\left(t_{0}\right)+F_{\text {wave } x} d t=p_{\perp}(\eta) \\
& p_{y}\left(t_{0}\right)+F_{\text {wave } y} d t=p_{\|}(\eta) .
\end{aligned}
$$

The important feature of the functions $p_{\perp}$ and $p_{\|}$is that they depend on $E$ and $\omega$ only through the ratio $E / \omega$, which is a Loreniz invariant, as explained above. 
Now consider the electron momentum in the frame moving with velocity $\mathrm{dv}$ relative to the original frame. In this frame the electron has momentum components

$$
\begin{aligned}
& p_{x}^{\prime}=p_{x}-m \gamma d v_{x} \\
& p_{y}^{\prime}=p_{y}-m \gamma d v_{y},
\end{aligned}
$$

where $\gamma$ is the Lorentz factor of the electron in the original frame. In the primed frame, however, the $x$ axis, which is parallel to the original $x$ axis, is no longer parallel to the wave axis, because the wave direction changes under Lorentz transformations. Assuming $d t$ taken small enough that

$$
\mathbf{F}_{\mathrm{ext}} d t \sim \mathrm{dv}_{d} \ll c
$$

the wave in the new frame is altered by a rotation through an angle $d v_{y} / c$. This result (the aberration of light) can be derived either from the transformation of the electric and magnetic fields or from the velocity addition formulas, treating the wave as a particle moving with the speed of light. ${ }^{15}$ The frequency of the wave is also changed, so the amplitude of the oscillations is changed, but the relationship between momentum and phase depends only on $a$ and is hence preserved.

Fig. 1 shows the figure-eight motion from the same plane wave in two different frames, one the lab frame (in which the wave travels in the $x$ direction) and the other moving in the positive $y$ direction with velocity $0.5 c$. The angle of rotation satisfies $\sin \theta=d v_{y} / c$, and (to first order in $\left.d v_{y} / c\right) \cos \theta=1$. From this rotational effect,

$$
\begin{aligned}
& p_{\|}^{\prime}=p_{x}^{\prime}+\frac{p_{y}^{\prime} d v_{y}}{c}=p_{x}-m d v_{x} \gamma+\frac{p_{y}^{\prime} d v_{y}}{c} \\
& p_{\perp}^{\prime}=p_{y}^{\prime}-\frac{p_{x}^{\prime} d v_{y}}{c}=p_{y}-m d v_{y} \gamma-\frac{p_{x}^{\prime} d v_{y}}{c} .
\end{aligned}
$$

Our goal is to choose $d v_{x}$ and $d v_{y}$ so that the electron is at rest on average in the frame moving with velocity $\mathbf{d v}$. In other words,

$$
\begin{aligned}
& p_{\|}^{\prime}=p_{\| \Uparrow}(\eta) \\
& p_{\perp}^{\prime}=p_{\perp}(\eta) .
\end{aligned}
$$

Combining equations (3), (4), (5), (7), and (8), and writing $d p$ for $\mathbf{F}_{\text {ext }} d t$, we obtain

$$
\begin{aligned}
& p_{\|}(\eta)+d p_{x}-m \gamma d v_{x}-\frac{m p_{\perp}(\eta) d v_{y}}{c}=p_{\|}(\eta) \\
& p_{\perp}(\eta)+d p_{y}-m \gamma d v_{y}+\frac{m p_{\|}(\eta) d v_{y}}{c}=p_{\perp}(\eta) .
\end{aligned}
$$


In these equations we have dropped terms of order $d v^{2} / c^{2}$, since these terms make no contribution in the limit $d t \rightarrow 0$. Related terms will return, however, when we attempt to sum the infinitesimal impulses from the background field. Solving the above equations, we obtain

$$
d v_{x}=\frac{d p_{x}}{m \gamma}-\frac{p_{y} d p_{y}}{m \gamma\left(m c \gamma-p_{\|}(\eta)\right)}, \quad d v_{y}=\frac{c d p_{y}}{m c \gamma-p_{\|}(\eta)}
$$

We can simplify these equations slightly by noting that $\gamma-p_{\|}(\eta) / m c$ is a constant (called $\gamma$ by Landau and Lifshitz, henceforth $\gamma_{0}$ ) with value $\sqrt{1+a^{2} / 2}$, where $a$ is the strength parameter of the wave defined above. The phase-averaged (not time-averaged) relativistic mass of an oscillating electron is $\gamma_{0}$, and the reciprocal of the time average of the reciprocal of $\gamma$ for an oscillation is also $\gamma_{0}$. This important relationship follows from the third invariant of the unperturbed motion:

$$
\gamma-\frac{p_{\|}}{m c}=\gamma_{0} \Rightarrow \frac{\gamma_{0}}{\gamma}=1-\frac{p_{\|}}{m c \gamma}=1-\frac{v_{\|}}{c}=\frac{d \eta}{d t}
$$

Therefore averaging $1 / \gamma$ with regard to time is equivalent to averaging $1 / \gamma_{0}$ (a constant) with regard to $\eta$. For circular polarization $p_{\|}$is zero and $\gamma=\gamma_{0}$. For linear polarization, the time average of $p_{\|}$is nonzero in the rest frame, where $v_{\|}$averages to zero; this occurs because the variation of $\gamma$ is correlated with that of $p_{\|}$. The statement that the electron has zero average momentum in the rest frame is thus not strictly correct; the rest frame should be defined as the frame with zero average velocity, in cases where the two are not identical. The calculations for the $z$ direction are the same as those for the $y$ direction, so finally we obtain

$$
d v_{y}=\frac{d p_{y}}{m \gamma_{0}}, \quad d v_{z}=\frac{d p_{z}}{m \gamma_{0}}, \quad d v_{x}=\frac{d p_{x}}{m \gamma}-\frac{p_{y} d p_{y}}{m^{2} c \gamma \gamma_{0}}-\frac{p_{z} d p_{z}}{m^{2} c \gamma \gamma_{0}}
$$

writing $p_{y}$ and $p_{z}$ for the two transverse components of the exact solution for the momentum (which are equal to the $y$ and $z$ momenta in the original frame neglecting the external force, or to the $y$ and $z$ momenta in the new frame if we take into account the rotation required by the aberration of light).

The equations (12) hold for arbitrary polarization and for any wave causing periodic motion. The equations and the approximation used to derive them are accurate for arbitrary $a$; however, in deriving them we made the assumption that the total velocity gain from the impulse was much less than $c$. The form given above is useful for collisions and other short-time-scale behaviors, but we 
can extend it without great difficulty if we retain the assumption that the total momentum supplied by the external forces is insufficient to make the drift velocity relativistic (the quiver velocity, of course, remains arbitrarily relativistic). Note that the velocity gains are separately linear in each of the components of the applied force.

There are three effects of order $v_{d} / c$ smaller than the leading velocity gain we wish to calculate. Including these effects complicates the equations sufficiently that numerical solution seems to be the only means of future progress. Velocity gains from a continuous series of impulses can be added simply (i.e., linearly) if we know that the sum of the magnitudes of the velocities is much less than c, but relativistic addition of velocities introduces second-order terms that become comparable as $v_{d}$ nears $c$. As described above, the perceived change in the wave's direction of incidence has the effect of a rotation of coordinates. This rotation means that the wave's direction of incidence in the drift frame is no longer parallel to $x$, but the error arising from ignoring this rotation is of order $v_{d} / c$ smaller than the result of the first-order calculation (since the rotation angle scales as $\left.v_{d} / c\right)$. Finally, the background electric and magnetic fields in the drift frame differ from those in the original frame by a factor of order $v_{d} / c$. The assumption that the drift velocity remains non-relativistic under small perturbations is justified by the impulsive equations above. Ignoring these three corrections, we obtain the differential equations for the drift velocity

$$
\frac{d v_{y}}{d t}=\frac{F_{y}}{m \gamma_{0}}, \quad \frac{d v_{z}}{d t}=\frac{F_{z}}{m \gamma_{0}}, \quad \frac{d v_{x}}{d x}=\frac{F_{x}}{m \gamma}-\frac{F_{y} \nu_{y}}{m c \gamma_{0}}-\frac{F_{z} \nu_{z}}{m c \gamma_{0}}
$$

where $\nu_{y}$ and $\nu_{z}$ represent the quiver velocity in the $y$ and $z$ directions rather than the drift velocity.

For many background fields the differential equations can effectively be averaged over a period to give the resulting drift acceleration. For example, for a uniform electric field $E$ along $\hat{x}$ we obtain that the drift velocity after one period is just $e E / m \gamma_{0} \omega$ (which only approaches $c$ for $E \sim 10^{10}$ $\mathrm{V} / \mathrm{cm}$ ) along $\hat{x}$. For sufficiently weak and uniform fields, however, it will be shown that the second and third terms in the $v_{x}$ equation vanish and the $\gamma$ in the first term can be averaged to $\gamma_{0}$. In this case the equation becomes simply

$$
\frac{\mathbf{d v}_{d}}{d t}=\frac{\mathbf{F}}{m \gamma_{0}}
$$


Equation (13) and the equation for the drift motion (14) are a major result of this paper; we now turn to the conditions of applicability of these equations and the errors caused by the use of the averaged motion (Section III), the extension of these equations to various types of background fields (Sections IV and V), and generalization of these equations to the case where the wave fields are changing in time (Section VI). Section VI also contains a frame-invariant version of the guidingcenter equation.

The impulse equations contain an interesting asymmetry between the $x$ response and the $y$ response, in that any impulse acting in the $y$ direction accelerates the electron as if it had mass $m \gamma_{0}$, rather than $m \gamma$, the effective mass in the $x$ direction. The effect of $d p_{y}$ on the $x$ motion is also surprising. These two effects are observed in a single-particle numerical simulation of the impulse problem. For uniform fields the asymmetry between $x$ and $y$ disappears. However, it is this asymmetry that explains the ponderomotive acceleration along the wave axis, which is examined in detail in Section VI.

\section{CONSTRAINTS ON THE GUIDING-CENTER DESCRIPTION}

This section examines the conditions under which (13) is valid, and the following two sections look at the conditions under which equation (13) can be averaged to (14). As we show, there are a number of subtleties in the use of these equations. First, note that the use of the averaged acceleration in place of the actual acceleration introduces an error, as in the following example. For an electric field along $y$, the drift velocity in the $y$ direction after a period is $e E / m \gamma_{0} \omega$, and the drift velocity in the $x$ direction is

$$
\int_{\text {period }} \frac{-e E \nu_{y} d t}{m c \gamma_{0}}
$$

This integral is zero to our current level of approximation, so that there is no velocity gain over a period in the $x$ direction. However, the integral of the drift velocity over a period ("the drift displacement") car be nonzero, depending on when the period is taken to begin, since the electron may gain drift velocity in one direction and then lose it, resulting in zero net velocity gain but in some position gain. Fig. 2 shows the acceleration, velocity, and displacement graphs in the $x$ 
direction for an applied field along $y$ that is turned on instantly at one point in the oscillation. We have

$$
v_{x}(t)=\int_{0}^{t} \frac{-e E \nu_{y} d t}{m c \gamma_{0}}=-\frac{e E y(t)}{m c \gamma_{0}}
$$

where $y(0)$ is chosen to be 0 and $y(t)$ represents the electron's displacement within its figure-eight orbit rather than relative to the lab. If, say, at time 0 the electron is at its highest $y$ displacement, then for all times the velocity in the $x$ direction will be in the direction $e E$, although this velocity is periodic in the same way as $y(t)$. Using the equation given below for the function $y(t)$, the integration gives an average velocity in the $x$ direction of magnitude

$$
v_{x}=\frac{2 e E y_{\max }}{m c \gamma_{0}}=\frac{2 a^{2} c}{\gamma_{0}^{2}} \frac{E}{E_{0}} .
$$

This is one example of a systematic error resulting from the use of the average acceleration as an actual acceleration. In effect, information about the initial phase of the electron is lost in the transition to averaged "guiding-center" equations. The use of the averaged acceleration as the actual acceleration necessarily gives the correct final velocity, taken over an integral (or very large) number of periods; the final displacement may be inaccurate, as in the example given above. Often this "phase velocity" is of little interest, for two reasons. The first is that often the resulting velocity is less than the change in the drift velocity over a single period; since the drift velocity accumulates over many periods while the phase velocity does not, the drift velocity over practical times is much larger. As an example, consider the case with $a \gg 1$ and an applied electric field in the $y$ direction. Then

$$
v_{x}=\frac{4 c E}{E_{0}}, \quad v_{y}=\frac{\sqrt{2} \omega c t E}{E_{0}}
$$

and after a few periods $v_{y}$ is much larger than $v_{x}$. This argument does not hold for the case of a uniform magnetic field or other field which produces no drift acceleration averaged over a period; in this situation the phase velocity can dominate. The other reason the phase velocity can typically be neglected is that in practice either the applied field or the wave field is turned on over many oscillations. If the rise of the wave or electric field is uncorrelated with the wave frequency, then the effect of the phase velocity becomes much smaller. This happens for the same reason that a smooth wave packet (i.e., one which is effectively linear over a period) tends not to 
produce a significant displacement in the transverse direction: because the acceleration oscillations are changing in amplitude, the velocity changes sign after each period and oscillates with roughly zero average. As an example, note that

$$
\left\langle\int_{0}^{t} a \sin t^{\prime} d t^{\prime}\right\rangle=\langle a-a \cos t\rangle=\frac{a}{2}
$$

so that the phase velocity is nongero, while for a linearly rising wave packet

$$
\left\langle\int_{0}^{t} t^{\prime} \sin t^{\prime} d t^{\prime}\right\rangle=\langle\sin t-t \cos t\rangle \sim 0 .
$$

The average of $t \cos t$ is effectively zero, in that it remains bounded while the wave amplitude grows without bound.

Averaged over all initial phases, the phase velocity vanishes, for otherwise it would contribute to the acceleration. This can be shown as follows: if $a(t)$ is the acceleration producing the phase velocity at time $t$, define a function $v(t)$ by

$$
v(t)=\int_{0}^{t} a(t) d t
$$

Because the average of $a(t)$ is zero, $v(t)$ must be a periodic function with period $2 \pi / \omega$. Then for the averaged phase velocity we have

$$
\begin{aligned}
\langle v\rangle & =\left(\frac{\omega}{2 \pi}\right)^{2} \int_{0}^{2 \pi / \omega} d t_{0} \int_{t_{0}}^{t_{0}+2 \pi / \omega} d t \int_{t_{0}}^{t} a(t) d t^{\prime} \\
& =\left(\frac{\omega}{2 \pi}\right)^{2} \int_{0}^{2 \pi / \omega} d t_{0} \int_{t_{0}}^{t_{0}+2 \pi / \omega}\left(v(t)-v\left(t_{0}\right)\right) d t \\
& =\left(\frac{\omega}{2 \pi}\right)^{2} \int_{0}^{2 \pi / \omega} d t_{0} \int_{t_{0}}^{t_{0}+2 \pi / \omega} v(t) d t-\frac{\omega}{2 \pi} \int_{0}^{2 \pi / \omega} v\left(t_{0}\right) d t_{0} \\
& =\frac{\omega}{2 \pi} \int_{0}^{2 \pi / \omega} v(t) d t-\frac{\omega}{2 \pi} \int_{0}^{2 \pi / \omega} v\left(t_{0}\right) d t_{0}=0
\end{aligned}
$$

where in the next-to-last step we have used the periodicity of $v$.

The use of the averaged acceleration eliminates many of the terms in the equations for the drift motion, and the remaining terms closely resemble those for a non-oscillating electron with increased mass, as will be shown below. If the velocity gained by an electron is small over a single period, we can describe the behavior of the electron over many periods by using the acceleration averaged 
over a period as the electron's acceleration, and then treating this acceleration relativistically; this method will give the correct answer as long as the velocity gain over a period remains small, and will continue to describe the motion correctly as the aggregate drift velocity becomes relativistic. In some cases the secondary terms in $v_{x}$ do not vanish and make a necessary contribution, as in the case of a ponderomotive force associated with the growth in intensity of the original wave, which will be examined below. It will be shown that for many types of background fields, the electron motion is given to a high degree of approximation by a simple equation that effectively sums the contributions from the background fields and the wave's ponderomotive force.

One condition already mentioned that must be satisfied for the drift equations to be valid is that the drift velocity gain induced by an external field over a single period of the wave be nonrelativistic. If the drift velocity gain is relativistic, then the simple addition of velocities breaks down and transfer of energy from the wave to the electron or vice versa is possible. In more precise language, the addition is legal, and the motion described by the equations (13), if in the frame where the electron has no drift velocity the applied fields satisfy

$$
a_{\text {applied }}=\frac{e E_{\text {applied }}}{m c \omega_{\text {wave }}}=\frac{E_{\text {applied }}}{E_{\text {crit }}} \ll 1
$$

Here $e E_{\text {crit }}=m c \omega_{\text {wave }}$. ( $B_{\text {applied }}$ can be substituted for $E_{\text {applied }}$ in the above). Note that this constraint has nothing to do with the strength of the wave, and the wave can be weaker than the applied field, as long as the applied field is sufficiently weak. Strong background fields are numerically observed to produce very complicated motions, although certain regularities seem to exist in some cases.

As an example of the importance of this constraint, consider the accelerator scheme of Kawata et al. ${ }^{5}$, whereby a transverse static electric field is used to convert wave energy into particle energy. The constraint must be violated to produce significant energy transfer. In the lab frame the static electric field has $E_{\text {applied }} / E_{\text {crit }}=2.18 \times 10^{-5}$, In the frame of the electron, however (which has inital velocity $0.9999 c), \omega_{\text {wave }}$ and $E_{\text {rel }}$ are decreased by a factor $2 \gamma \sim 141$, and $E_{\text {applied }}$ is increased by a factor of $\gamma$. Thus in the electron's frame $E_{\text {applied }} / E_{\text {crit }} \sim 0.212$, and the absorption of wave energy by the electron does not contradict the above results. The results of our numerical simulation for these parameters match those in this paper. In another paper ${ }^{6}$ Kawata et al. use a magnetic field 
with $a_{\text {applied }}=0.0057$ in the lab frame and initial $\gamma$ of 3.2 , so that $2 \gamma^{2} a_{\text {lab }}=a_{\text {drift }}=0.117$. In fact, the correct $\gamma$ to use in calculating $a_{\text {drift }}$ should include the ponderomotive increase in the electron drift velocity, which will be discussed in greater detail below; the actual $a_{\text {drift }}$ values for the two methods described above are 0.243 and 0.133 . In another acceleration method, that of Apollonov et al. ${ }^{4}$, the prescribed initial $\gamma$ is equal to $(\omega m c / 2 e B)^{1 / 2}$, which is exactly our condition that $2 \gamma^{2} B \sim E_{\text {crit }}$.

If an applied electric field is weak, then according to the above equations the drift acceleration of the particle can never be more than twice as large as the acceleration of a stationary electron in the same field ("twice" because of the additional terms in the $x$ equation) and will typically be less because of the increase in effective mass. A weak applied magnetic field that is highly nonuniform over a figure-eight can lead to significant acceleration, and this case is considered below. For simplicity, the rest of the results in this paper will be specific to the case of linear polarization unless otherwise noted. The generalization to other polarizations is in most cases straightforward.

\section{SIMPLE APPLICATIONS OF THE EQUATION}

Some results regarding simple types of background fields can be easily determined from the equations above. For simplicity, these results will be derived for the case of linear polarization; in most cases the treatment for arbitrary polarization is similar, although the results may differ. The unperturbed equations of motion in the rest frame of the electron, for the vector potential $\mathbf{A}=A_{y}=-a m c^{2} \sin (\eta) / e, a r e^{12}$

$$
\begin{gathered}
x=-\frac{a^{2} c \sin 2 \eta}{8 \gamma_{0}^{2} \omega}, \quad y=-\frac{a c \cos \eta}{\gamma_{0} \omega}, \quad z=0, \\
p_{x}=p_{\|}=-\frac{a^{2} m c \cos 2 \eta}{4 \gamma_{0}}, \quad p_{y}=p_{\perp}=-a m c \sin \eta, \quad p_{z}=0 .
\end{gathered}
$$

As described above, the drift induced by a uniform electric field is in the direction of the electric field, and the electron's effective mass is $m \gamma_{0}$. For a uniform magnetic field, the equations of motion (13) give the integrals

$$
\Delta v_{y}=\int \frac{-e \nu_{x} B_{z}}{m c \gamma_{0}} d t, \quad \Delta v_{z}=\int \frac{e\left(\nu_{x} B_{y}-\nu_{y} B_{x}\right)}{m c \gamma_{0}} d t, \quad \Delta v_{x}=\int \frac{e \nu_{y} B_{z}}{m c \gamma}-\frac{-e \nu_{x} B_{z}}{m \gamma_{0} c} \frac{\nu_{y}}{c} d t
$$


Since $\int v_{i} d t=0$ for each index $i$, the first two integrals are zero. The $x$ integral is also zero, as can be verified by direct computation or by use of some symmetries of the figure-eight motion that will be described below. As a result, a uniform magnetic field in the rest frame of the electron has no effect to first order in the strength of the magnetic field. For large magnetic fields the motion ceases to oscillate in an orderly manner.

This result can be used to explain the behavior of drifting figure-eight orbits in a uniform magnetic field. Suppose for example that a laser pulse induces (through the ponderomotive force) a drift velocity relative to the lab frame. During the body of the pulse, figure-eights moving with this drift velocity then move in cyclotron orbits in the presence of weak magnetic fields. In order to get these effects from the equations above, we begin by transforming the magnetic field from the lab frame to the electron's drift frame. The equations below are written for the case $\mathbf{v}_{d} \perp \mathbf{B}$; a parallel component of $\mathbf{v}_{d}$ merely makes the motion helical rather than circular. The fields in the drift frame are then $\mathbf{E}=\gamma_{d} \mathbf{v}_{d} / c \times \mathbf{B}$ and $\mathbf{B}=\gamma_{d} \mathbf{B}$. Here $\gamma_{d}$ is the Lorentz factor of the drift motion. The drift velocity of the figure-eight in the lab frame can be arbitrarily large without causing difficulties for the method described above, as long as the change in drift velocity over a period is nonrelativistic. In this moving frame, then, the electron experiences an acceleration perpendicular to the direction of its drift velocity with magnitude $e \gamma_{d} v_{d} B / m c \gamma_{0}$. The assumption that the fields are effectiveiy constant over a period in the drift frame requires that $\omega_{c} \ll \omega_{\text {wave }}$, but this criterion is difficult to violate for wavelengths of interest. Essentially the criterion states that the fields in the electron's trift frame change slowly over a period of the wave. Transforming a perpendicular acceleration introduces two time dilation factors of $\gamma_{d}$, so that in the lab frame the electron experiences an acceleration $e v_{d} B / m c \gamma_{0} \gamma_{d}$. Hence the modified Larmor frequency $\omega_{c}$ is $e B / m c \gamma_{0} \gamma_{d}$, and this result is observed numerically.

The multiplication of Lorentz factors in the denominator of the previous expression can be understood by viewing the oscillating electron as a "quasiparticle" of mass $m \gamma_{0}$. The transformations to and from the drift frame in the above calculation can be generalized simply by noting that a particle whose displacement satisfies the Lorentz equation of motion in one frame is constrained to obey it in all frames. Thus we have the general result that, for fields which in the rest frame 
of the electron vary slowly over the single-oscillation time and space scales and which do not induce relativistic velocities over a period of the wave, the electron behaves like a quasiparticle of enhanced mass $m \gamma_{0}$. This enhanced mass, and its effect on the plasma frequency, has appeared in the literature before, for example in studies of focussing of laser beams by plasmas ${ }^{16}$; the complete derivation here of the enhanced mass and the conditions for its applicability, however, appear to be new. The methods described here can also be applied for weak fields which are not uniform in space or time, as in the following examples.

Although figure-eights make closed cyclotron orbits to first order, this analysis cannot rule out a velocity change of order $\left(d v_{\text {period }}^{2} / c\right)$ per oscillation or $\left(\omega / \omega_{c}\right)\left(d v_{\text {period }}^{2} / c\right)$ per cyclotron orbit. For sufficiently strong magnetic fields, even electrons with zero inital drift velocity are strongly affected, and the orbits no longer resemble cyclotron motion. Fig. 3 shows the progressive breakdown of the cyclotron motion; it is interesting that the motion retains some regularities even for applied fields of very high strength. When the electron has zero dirift velocity in a constant magnetic field, to first order the magnetic field causes no acceleration and the higher-order terms dominate. The methods given in this paper calculate what in mosi circumstances is the dominant part in the motion; in certain special cases the part calculated here goes to zero and other less easily determined behaviors become evident. The simplest, most important such case is that of a static uniform field which in the electron rest frame is purely magnetic.

\section{MOTION IN NONUNIFORM FIELDS}

$\rightarrow$

This section extends the conditions under which the asymmetry in the guiding-center equation disappears upon averaging. The results obtained in this section will depend on the linear polarization of the incident wave; the effects of varying fields on oscillations in circularly and elliptically polarized waves are much different. The primary result is that, for linear polarization, fields which vary linearly allow the averaging of equation (13), even if the fields vary significantly over a figure-eight length scale. Hence the guiding-center equations are applicable for a wider class of background fields if the wave is linearly polarized. 
A uniform magnetic field causes no change in the drift velocity over a period, as shown in the previous section. In the presence of a spatially varying magnetic field, we expect a drift acceleration of order

$$
a_{\text {grad }}=\frac{e v_{\text {osc }} \lambda \nabla B}{m c}
$$

where $\lambda$ is used as an estimate of the figure-eight excursion distance. If the electron is moving with velocity $v_{d}$ relative to the frame where the field is purely magnetic, the magnetic field (ignoring the gradient) induces an acceleration in the drift frame of magnitude

$$
a_{\text {drift }}=\frac{e v_{\text {drift }} \gamma_{\text {drift }} B}{m c}
$$

Comparing the two, we get

$$
a_{\text {grad }} \ll a_{\text {drift }} \Rightarrow \frac{\nabla B}{B} \lambda \ll \frac{v_{\text {drift }}}{v_{\text {osc }}} .
$$

Depending on the size of $v_{\text {drift }}$ and the degree to which the magnetic field varies over $\lambda$, the gradient may induce effects less than, comparable to, or larger than the effect of the field without the gradient. Because $v_{\text {osc }}$ can be relativistic while $v_{\text {drift }}$ is small, even for fields varying by a few percent on the oscillation scale the gradient term can be larger than the static term. For the case of circular or elliptical polarization, these estimates are substantially correct: fields varying slightly on the oscillation scale can invalidate the guiding-center picture and yield energy transfer from the wave to the particle. For linear polarization, however, linearly varying fields end up causing a much smaller change in the acceleration. Magnetic fields are of primary interest because a gradient in an applied electric field changes the acceleration by at most a factor of $\nabla E / E$, so that the effect is small unless the electric field changes on a scale length less than or equal to a period.

The figure-eight motion in a linearly polarized wave has certain symmetries, and these symmetries greatly simplify the calculation of drift motions. Looking at the equations (24) we see that $p_{x}$ has the same value at $\eta=\theta, \eta=\pi-\theta, \eta=\pi+\theta, \eta=2 \pi-\theta$ for any angle $\theta$. Similarly $p_{y}$ has the same value at $\eta=\theta$ and $\eta=\pi-\theta$, and the negative of this value at $\eta=\pi+\theta$ and $\eta=2 \pi-\theta$. Since the Lorentz factor $\gamma$ is equal to $\sqrt{1+\left(p_{x} / m c\right)^{2}+\left(p_{y} / m c\right)^{2}}$, we also have that $\gamma$ takes on the same value at the four phase angles. These results are significant because the positions at the four phase angles in question form a rectangle, and for linear field gradients the sum of the field at the four 
vertices of a rectangle takes a particularly simple form. The four phase angles $\theta, \pi-\theta, \pi+\theta, 2 \pi-\theta$ correspond to the top left, bottom right, bottom left, and top left corners of the rectangle, as drawn in Fig. 4. These facts are summarized in the following table:

\begin{tabular}{|c|c|c|c|}
\hline Phase & $p_{x}$ & $p_{y}$ & $\gamma$ \\
\hline$\theta$ & + & + & + \\
$\pi-\theta$ & + & + & + \\
$\pi+\theta$ & + & - & + \\
$2 \pi-\theta$ & + & - & + \\
\hline
\end{tabular}

In calculating the acceleration induced by an electric field, we obtain four integrals over the period of the motion, which can be done explicitly using (24). Two of these integrals are proportional to $\int \mathbf{E}(\mathbf{x}) d t$, one is proportional to $\int E_{x}(\mathbf{x}) / \gamma d t$, and one is proportional to $\int E_{y}(\mathbf{x}) \nu_{y} d t$. In the case of the first two types, the part of the integrand not depending on $\mathbf{E}$ takes on the same value at each of the four points on the rectangles described above. The original integrals are over time rather than phase, but they can be converted easily, noting that $d t / d \eta$ depends only on $v_{x}$ and hence has the same value at the vertices of one of the rectangles constructed above. If the electric field varies linearly with position, the value of $\mathbf{E}$, averaged over these four points, is equal to its value at the center. Therefore each of these integrals gives the same answer as if $\mathbf{E}$ were constant, with value equal to the value of the actual electric field at the figure-eight origin. In the last integral, however, the integrand takes on different values at the vertices of the rectangle: it takes on one value at the top left and bottom right corners, and the negative of this value at the other pair of opposite corners. As a result, for a linearly varying electric field, this last integral has value zero, the same value as it has for any constant electric field. Therefore, for a weak, linearly varying electric field, the acceleration over a period is the same as that of a particle of mass $m \gamma_{0}$ and charge $e$ in a constant field with magnitude equal to the magnitude of the actual field at the origin. The only interaction between the figure-eight and the field gradient occurs through the drift velocity.

The magnetic field case is only slightly more complicated; now the integrands contain magnetic field terms multiplied by $\nu_{x}, \nu_{y} / \gamma$, and $\nu_{x} \nu_{y}$. (Once again an additional factor of $d t / d \eta$ enters that does not affect the calculation) The second two integrands thus average to zero for linearly varying fields, and the first gives the same effect as that of a uniform magnetic field with value at the origin 
equal to the value of the original field at the origin. Integrating $\nu_{x}$ over a period (for a uniform magnetic field) gives zero, however, so that magnetic gradients have no effect. The gradients affect the motion only through the drift velocity, therefore, so that figure-eights in linearly varying magnetic fields should exhibit $B \times \nabla B$ drifts similar to those of electrons not in waves. This behavior is observed numerically (Fig. 5): oscillating electrons in a linearly varying magnetic field drift with velocity

$$
v_{g}=\frac{m v_{\perp}^{2} \gamma_{0} \gamma_{\mathrm{drift}}}{2 e B} \frac{\nabla B}{B}
$$

The above calculation only considers the interaction of the figure-eight motion with the magnetic field (because the integration was performed over the unperturbed motion); as the drift velocity of the particle increases over a period, the position of the particle measured in the original drift frame no longer lies exactly on the original figure-eight. This effect is smaller by a factor $\Delta v_{d} / v_{\text {quiver }}$ than the term calculated above, and can on'y be comparable to the acceleration induced by a static field of equal magnitude if $\Delta v_{d} \nabla B / \omega \sim v_{d} B$, i.e., the electron is stationary or the scale length is less than a wavelength. It should be pointed out that a spatially varying field in the lab frame may vary in time as well as space in the electron's drift frame, and that even linear time variation can cause an additional acceleration. This acceleration is purely phase-dependent, however, and therefore has a negligible effect unless the electron's drift velocity changes greatly within a period.

For magnetic fields which are weak but vary nonlinearly on the figure-eight scale, there can be significant uptake of energy by the electron (i.e., transfer of oscillation energy to drift energy). The simplest example is a field which is nonzero for only a small part of the orbit; then the figure-eight feels a large drift acceleration over this portion of the oscillation.

\section{PONDEROMOTIVE FORCE IN PRESENCE OF EXTERNAL FIELDS}

To this point the analysis has been specific to the case of plane waves of constant amplitude. Waves of varying amplitude (e.g., pulses) generate the well-known ponderomotive force in the direction of motion. The ponderomotive force in the absence of background fields can be easily derived from the three invariants of the motion. We present a calculation of the ponderomotive force using a variation of the frame-transformation method applied above to demonstrate that 
the ponderomotive force is unchanged when background fields are present, under a suitable set of assumptions. We then derive a covariant equation of motion that describes the behavior of an electron in an arbitrarily intense but "smooth" pulse, in the presence of weak background fields. The ponderomotive force results from a time-dependent scalar potential that happens to be conservative in the absence of external fields, i.e., after the wave has passed the electron has its initial energy. An important consequence of these equations is that energy can be transferred to an electron by the ponderomotive force if background fields are present.

The ponderomotive force derives from a change in the functions $p_{\|}$and $p_{\perp}$ over time, resulting from the change in the wave amplitude $a$ over time. We use $a$ to denote the envelope amplitude, rather than the instantaneous amplitude. Writing $\Delta p$ for the change in rest-frame momentum resulting from the change in the wave amplitude, our goal is to find the velocity $d v$ of the frame in which the electron has parallel momentum $p_{\|}+\Delta p_{\|}$and perpendicular momentum $p_{\perp}+\Delta p_{\perp}$. By the same steps that led to (9), but with no external force and the momentum in the primed frame altered by $\Delta p$,

$$
p_{\|}-m d v_{x} \gamma-\frac{p_{\perp} d v_{y}}{c}=p_{\|}+\Delta p_{\|}, \quad p_{\perp}-m d v_{y} \gamma+\frac{p_{\|} d v_{y}}{c}=p_{\perp}+\Delta p_{\perp}
$$

Therefore the effect of an amplitude change is equivalent in our formalism to a force of $-d p_{\|} / d t$ in the $x$ direction and $-d p_{\perp} / d t$ in the $y$ direction (the negative signs appear because the force terms are on the left in (9)). The derivatives with respect to time should reflect only the change in wave amplitude, i.e., $-d p_{\|} / d t$ should be properly written $\left(-\partial p_{\|} / \partial a\right)(d a / d t)$, and similarly for the $y$ direction. Since the ponderomotive force enters just as any other force, and the equations for the drift velocity are linear in the applied force, it follows that the ponderomotive force does not interact (for short times) with any other force that may be present if both are sufficiently weak. This result is nontrivial because the ponderomotive "force" is ordinarily derived from the three constants of the motion in the unperturbed case; since these constants are not preserved, there is no guarantee that the ponderomotive force should take a similar form. We have again the equations of motion

$$
\frac{d v_{y}}{d t}=\frac{F_{y}}{m \gamma_{0}}, \quad \frac{d v_{x}}{d x}=\frac{F_{x}}{m \gamma}-\frac{F_{y} \nu_{y}}{m \gamma_{0}}
$$


The ponderomotive velocity reached as a wave rises depends only on the final amplitude of the wave and the final value of the vector potential, but this simplicity is obscured in the above equations, in that the ponderomotive force can come from any of the three terms in the equation above. We will concentrate on the case when the wave rises slowly, i.e., over many periods and at an approximately constant rate over each period; in this case the $y$ equation is zero on average, so that the ponderomotive force is directed along the wave axis. The averaging of the $x$ equation over a period, using the explicit equations (24) for the figure-eight motion, is not difficult (both terms contribute, however; see $A$ sppendix $A$ ) and yields for the average acceleration

$$
\left\langle\frac{d v_{x}}{d t}\right\rangle=\frac{a c w}{2 \gamma_{0}^{2}} \frac{d a}{d \eta}
$$

This equation is in the rest frame of the electron, but note that $d a / d \eta$ is invariant, as both $a$ and $\eta$ are relativistic invariants. Integration of this equation in its present form is difficult, since the (possibly relativistic) velocity of the rest frame complicates addition of velocities. Later we will show that the equations can be written in an easily integrable form in the absence of external fields.

We thus have an expression for the ponderomotive force in the frame where the electron has no drift velocity, as well as our previous expression for the behavior of the drift velocity in the presence of certain types of background fields. That is, in the drift frame we have

$$
m \gamma_{0} \frac{\mathbf{d v}_{d}}{d t}=e\left(\mathbf{E}+\mathbf{v}_{d} \times \mathbf{B}\right)+\frac{a m c \omega \hat{\mathbf{k}}}{2 \gamma_{0}} \frac{d a}{d \eta}
$$

These equations can be made frame-independent without great difficulty and take a more intuitive form. First we note that by forming the number $\gamma_{d}$ defined as $1 / \sqrt{1-v_{d}{ }^{2} / c^{2}}$, the vector $\nu_{d}=$ $\left(\gamma_{d}, \gamma_{d} \mathbf{v}_{d} / c\right)$ is a four-vector. This four-vector represents the time-averaged four-velocity if all background forces and ponderomotive effects vanished; this vanishing is a frame-invariant concept, and the average of a four-vector along a path in spacetime parametrized by a quantity transforming linearly (in this case the time) is a four-vector. This four-vector has constant length 1 , clearly. The equation (33) generalizes naturally to

$$
m \gamma_{0} \frac{d \nu_{d}^{\alpha}}{d \tau}=e F_{\beta}^{\alpha} \nu_{d}^{\beta}+(\text { ponderomotive term })
$$


Here $F_{\beta}^{\alpha}$ is the electromagnetic field tensor. For the ponderomotive term, we need a four-vector which is parallel to the vector $(0, \hat{k})$ when $\nu_{d}$ is equal to $(1,0,0,0)$. The ponderomotive fourvector must also be orthogonal to $\nu_{d}$, since $\nu_{d}$ has constant length (this condition is satisfied for the electromagnetic term by the skew-symmetry of $F$ ). The simplest four-vector satisfying these conditions is proportional to $\omega^{\alpha}-\nu_{d}^{\alpha}\left(\omega^{\beta} \nu_{d \beta}\right)$ where $\omega=(\omega, \omega \hat{k})$ is the wave four-vector. In order to make our equation look more like a force equation, introduce the four-vector $p_{d}$ defined as $m c \gamma_{0} \nu_{d}$ (the time-averaged momentum four-vector, if all background fields disappear). By the chain rule

$$
\frac{d p_{d}^{\alpha}}{d \tau}=m c \nu_{d}^{\alpha} \frac{d \gamma_{0}}{d \tau}+m c \gamma_{0} \frac{d \nu_{d}^{\alpha}}{d \tau}
$$

The second term is given by (34); the first is

$$
m c \nu_{d}^{\alpha} \frac{d \gamma_{0}}{d \tau}=\frac{a m c \nu_{d}^{\alpha}\left(\omega^{\beta} \nu_{d \beta}\right)}{2 \gamma_{0}} \frac{d a}{d \eta}
$$

This term cancels part of the first term, since

$$
\left(\omega^{\alpha}-\nu_{d}^{\alpha}\left(\omega^{\beta} \nu_{d \beta}\right)\right)+\nu_{d}^{\alpha}\left(\omega^{\beta} \nu_{d \beta}\right)=\omega^{\alpha}
$$

We are left with

$$
\frac{d p_{d}^{\alpha}}{d \tau}=e F_{\beta}^{\alpha} \nu_{d}^{\beta}+\frac{a m c \omega^{\alpha}}{2 \gamma_{0}} \frac{d a}{d \eta}
$$

which is a major result of this paper.

Because the ponderomotive term is parallel to $\omega^{\alpha}$, the momentum transfer from the ponderomotive effect is equal to that from a number of photon absorptions. McDonald ${ }^{17}$ derives similar results in the absence of background fields starting from this assumption, which is valid only in the case of a slowly rising wave. It is important to note that the conditions for applicability of this equation are frame-dependent: the fields must be weak and approximately uniform in the electron rest frame. We see that the momentum transfer per proper time varies between frames in the same way as $\omega^{\alpha}$, consistent with the photon picture: the number of absorptions is naturally invariant (a count of distinct spacetime events is preserved), but the characteristics of the photons absorbed change with $\omega^{\alpha}$. The rate at which momentum is instantaneously transferred to or from the wave depends only on the derivative $d a / d \eta$, an intrinsic property of the wave. The total momentum 
transferred, however, depends on the proper time spent by the electron at various points in the wave, which depends on the initial conditions.

This dependence can be demonstrated, and an easily integrable form of the equation obtained, by writing the ponderomotive term as

$$
\frac{a m c \omega^{\alpha}}{2 \gamma_{0}} \frac{d a}{d \tau} \frac{d \tau}{d \eta}=\frac{a m^{2} c \omega^{\alpha}}{2 p_{d}{ }^{\beta} \omega_{\beta}} \frac{d a}{d \tau}
$$

In the absence of fields other than those of the wave, the change in $p_{d}$ over time is parallel to $\omega^{\alpha}$, leaving the quantity $p_{d}{ }^{\beta} \omega_{\beta}$ constant. Therefore we can simply integrate both sides with regard to proper time and obtain an expression for the ponderomotive velocity. For example, if the electron is initially at rest, then $p_{d}{ }^{\beta} \omega_{\beta}$ has constant value $m \omega$, so that

$$
p_{d}=p_{0}+\frac{a^{2} m c}{4 \omega} \omega^{\alpha}
$$

and the drift velocity

$$
v_{d}^{x}=\frac{p^{x}}{p^{0}}=\frac{a^{2} c}{a^{2}+4}
$$

which is the correct result.

In the presence of external fields, even weak ones, the product $p_{d}{ }^{\beta} \omega_{\beta}$ is no longer constant, with interesting consequences. In the absence of external fields, it is well known that the net effect of the ponderomotive force over the pulse is just a displacement in the wave direction, but this no longer holds if an external field changes the denominator. As a simple example, a weak magnetic field combined with ponderomotive gradients can yield a significant change in energy, when neither of these two forces acting independently can change the energy at all. The momentum transfer from the ponderomotive force is largest when the momentum vector is parallel to $\hat{k}$, so that particles can be accelerated or decelerated in the wave direction by moving the momentum away from or toward $\hat{\mathbf{k}}$ during the body of the wave, respectively.

The ponderomotive terms in the above force equation can be derived from a potential

$$
V=-m c^{2} \gamma_{0}
$$

which is the negative of the phase-averaged energy of oscillation. The background field terms in (37) are identical to those for an ordinary charged particle. Since the ponderomotive term is the 
derivative of a function depending only on space and time (through $\eta$ ), the drift equations (37) are Hamiltonian with $B$ equal to the Hamiltonian for a charge in an electromagnetic field plus the ponderomotive potential term explained above. The ponderomotive potential is time-dependent and therefore the energy need not be conserved, even between times when the ponderomotive potential is the same (e.g., before and after the wave passes).

\section{SUMMARY}

For electromagnetic fields which are sufficiently weak and uniform in the rest frame of an oscillating electron, the presence of the wave affects the motion through an increase in the effective mass of the electron and through a ponderomotive force during periods when the wave amplitude is changing. The equation (37) describes the particle's motion under fairly general circumstances. Something that can be seen directly in this representation is that weak, uniform fields cannot induce significant energy transfer from a wave of constant amplitude to the electron or vice versa (no "inverse bremsstrahlung"), although a high electron velocity may make weak fields effectively many times stronger. Interaction between applied fields and the ponderomotive gradient of a wave can lead to some energy transfer in either direction. The motion of an oscillating electron for weak but not necessarily uniform fields is governed by the equations (13) for the drift velocity. We therefore have a nearly complete picture of single-particle behavior in the weak-field regime.

The general behavior of oscillating electrons in strong background fields presents a more difficult mathematical challenge. Computer simulation suggests that there are certain regularities in the motion, even for background magnetic fields strong enough to destroy the figure-eight motion. Some types of fields seem likely to induce stochasticity, however, e.g., two plane waves at incommensurable frequencies. An investigation of the case of multiple intense plane waves has been carried out by $\operatorname{Rax}^{11}$, but the general problem of intense background fields remains uncharted territory.

\section{ACKNOWLEDGEMENTS}

The authors wish to thank M. Herrmann and B. Chandran for helpful conversations.

This work was supported by the U.S. Department of Energy under Contract No. DE-AC0276-CHO3073. 


\section{APPENDIX A: RELATION OF GUIDING-CENTER VELOCITY TO DRIFT VE- LOCITY}

Our current definition of the drift velocity has the advantage of simplicity but does not represent an actual change in displacement over time; instead it represents the average displacement per time that would occur if the background fields were turned off. For some applications it might be more useful to deal with a guiding center or "instantaneous center of oscillation," defined as some point along the particle's orbit. For a figure-eight, we can conveniently define the center of oscillation as the crossing point of the figure-eight in which the electron is instantaneously moving. In other words, at any instant the electron is performing part of an oscillation in some drift frame, and the instantaneous center of oscillation is some point on this oscillation. As an analogy, in slowly varying magnetic fields electrons execute cyclotron orbits of varying radii, and it might be useful to operate with the center of the cyclotron orbit in which a drifting electron is inttantaneously moving. The center of oscillation moves for two reasons: the drift frame where it is located translates at the drift velocity $\mathbf{v}_{d}$, and the background fields change the amplitude and shape of the oscillation. To first order, the amplitude of oscillation is altered with $\omega$ and the shape is altered independently by the effective change in the incident direction. Suppose $\mathbf{x}(\eta)$ is a parametrization of the oscillation by the wave phase $\eta$, with origin at the (arbitrarily chosen) center of the oscillation. Once again eliminating terms of smaller orders, we obtain

$$
\begin{aligned}
\frac{\mathbf{d} \mathbf{x}_{o s c}}{d t} & =\mathbf{v}_{d}+\frac{\mathbf{d x}}{d \omega} \frac{d \omega}{d t}+\frac{\mathbf{d x}}{d \theta} \frac{d \theta}{d t} \\
& =\mathbf{v}_{d}-\frac{\mathbf{d x}}{d \omega} \frac{\omega d v_{x}}{c d t}-\left(\hat{\mathbf{k}} \times \frac{\mathbf{d} \mathbf{v}_{d}}{c d t}\right) \times \mathbf{x} .
\end{aligned}
$$

The last term on the right side of the above equation is bounded by $\lambda d v_{d} / c d t$, and integrating $d t$ gives that the contribution from this term is $\lambda v_{d} / c$, i.e., much smaller than a wavelength. Similarly the second term is bounded by $\lambda d v_{d} / c d t$, since $x$ scales with $1 / \omega$ if $a$ is kept constant. Thus we can treat $\mathbf{v}_{d}$ as the change in displacement of the oscillation center over time, with total error much smaller than a wavelength. One should keep in mind, though, that the effective wavelength of the pulse viewed in the electron frame may be rather large for highly relativistic electrons.

\section{APPENDIX B: CALCULATION OF PONDEROMOTIVE ACCELERATION}


The task is to integrate the differential equation for the drift velocity in the parallel direction over a period, using the exact solution to the motion (5). The two terms in the equation for $d v_{x} / d t$, averaged over a period, give

$$
\begin{aligned}
\left\langle\frac{d v_{x}}{d t}\right\rangle & =\frac{\omega}{2 \pi} \int_{0}^{2 \pi / \omega}\left(-\frac{1}{m \gamma} \frac{\partial p_{\|}}{\partial a} \frac{d a}{d t}-\frac{1}{m \gamma_{0}} \frac{\partial p_{\perp}}{\partial a} \frac{p_{\perp}}{m \gamma} \frac{d a}{d t}\right) d t \\
& =\frac{\omega}{2 \pi} \frac{d a}{d \eta} \int_{0}^{2 \pi}\left(-\frac{1}{m \gamma} \frac{\partial p_{\|}}{\partial a}-\frac{1}{m \gamma_{0}} \frac{\partial p_{\perp}}{\partial a} \frac{p_{\perp}}{m \gamma}\right) d \eta
\end{aligned}
$$

This integral takes a simple form when the exact solution (24) is substituted:

$$
\frac{\omega}{2 \pi} \frac{d a}{d \eta} \int_{0}^{2 \pi} \frac{(a c \cos 2 \eta) / 2+a c(1-\cos 2 \eta) / 2}{\gamma_{0} \gamma} d \eta=\frac{\omega}{2 \pi} \frac{d a}{d \eta} \int_{0}^{2 \pi} \frac{a c}{2 \gamma \gamma_{0}} d \eta
$$

The only variable term in the integrand is $\gamma$, and $1 / \gamma$ averages to $1 / \gamma_{0}$, as before. Therefore the average ponderomotive acceleration is

$$
\left\langle\frac{d v_{x}}{d t}\right\rangle=\frac{d a}{d \eta} \frac{a c \omega}{2 \gamma \gamma_{0}}
$$




\section{REFERENCES}

1. J. H. Eberly and A. Sleeper, Phys. Rev. 176, 1570 (1968); E. S. Sarachik and G. T. Schappert, Phys. Rev. D 1, 2738 (1970); T. W. B. Kibble, Phys. Rev. 150, 1060 (1966).

2. T. Tajima and J. M. Dawson, Phys. Rev. Lett. 4S, 267 (1979); C. Joshi and T. Katsouleas, eds., Laser Acceleration of Particles, AIP Conf. Proc. No. 130 (American Institute of Physics, New York, 1985); IEEE Trans. Plasma Sci. PS-15 (2) (1987).

3. P. Chen and J. M. Dawson, "The Plasma Wake Field Accelerator," in C. Joshi and T. Katsouleas, Laser Acceleration of Particles, AIP Conf. Proc. No. 130 (American Institute of Physics, New York, 1985); IEEE Trans. Plasma Sci. PS-15 (2) (1987).

4. V. V. Apollonov, A. I. Artem'ev, Yu. L. Kalackev, A. M. Prokhorov, and M. V. Fedorov, JETP Lett. 47, 91 (1988).

5. S. Kawata, T. Maruyama, H. Watanabe, and I. Takahashi, Phys. Rev. Letters 66, 2072 (1991).

6. S. Kawata, A. Manabe, S. Takeuchi, K. Sakai, and R. Sugihara, "Electromagnetic cross-field acceleration," in AIP Conference Proceedings 193: Advanced Accelerator Concepts, pp. 172-201, AIP, 1989.

7. C. I. Castillo-Herrera and T. W. Johnston, IEEE Transactions on Plasma Science 21, 125 (1993).

8. T. W. B. Kibble, Phys. Rev. 150, 1060 (1966).

9. C. E. Max, Phys. Fluids 18, 74 (1976); J. F. Lam, B. Lippman, and F. Tappert, Phys. Fluids 20, $1176(1977)$

10. M. O. Scully, Applied Physics B 51, 238 (1990).

11. Rax, J.-M., Phys. Fluids B 4, 3962 (1992).

12. V. I. Arnold, Ordinary Differential Equations, (MIT Press, Cambridge, Mass., 1978).

13. L. D. Landau and E. M. Lifshitz, The Classical Theory of Fields fourth revised English ed., p. 118 (Pergamon Press, London, 1975).

14. J. H. Eberly and A. Sleeper, Phys. Rev. 176, 1570 (1968).

15. (as 13), p.13 
16. P. Sprangle, C. M. Tang, and E. Esarey, IEEE Trans. Plasma Sci. PS-15, 2 (1987); X. L. Chen and R. N. Sudan, Phys. of Fluids B 5 (4) 1993.

17. K. T. McDonald, "Fundamental Physics During Violent Accelerations," in C. Joshi and T. Katsouleas, Laser Acceleration of Particles, AIP Conf. Proc. No. 130 (American Institute of Physics, New York, 1985) 


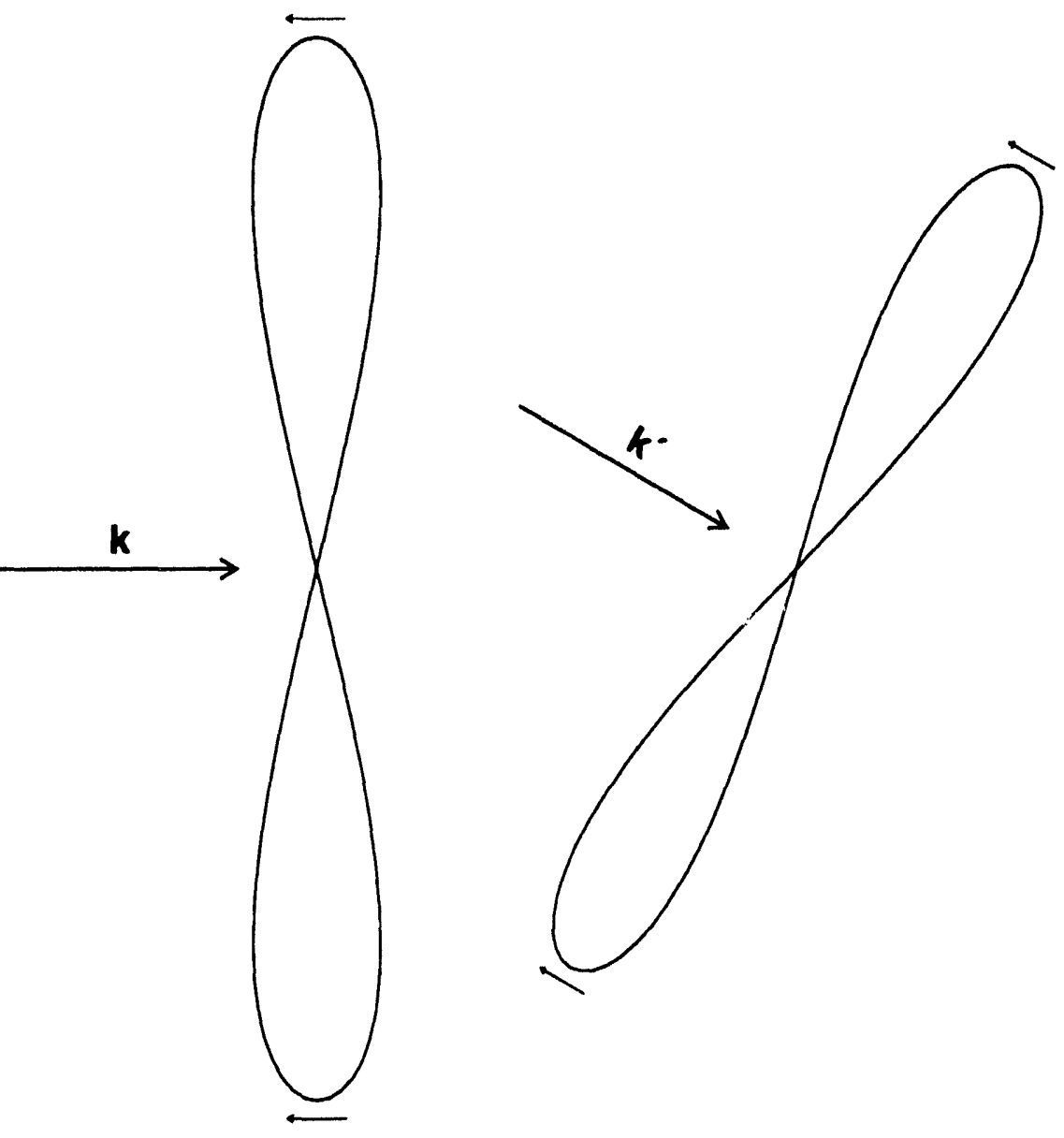

FIG. 1: Stationary oscillation in two frames. The right frame has velocity $0.5 c$ in the positive $y$ direction, measured in the left frame. As a result the figure-eight is reduced in size by a factor $\gamma=0.86$ and rotated by an angle $\theta=30^{\circ}$. 


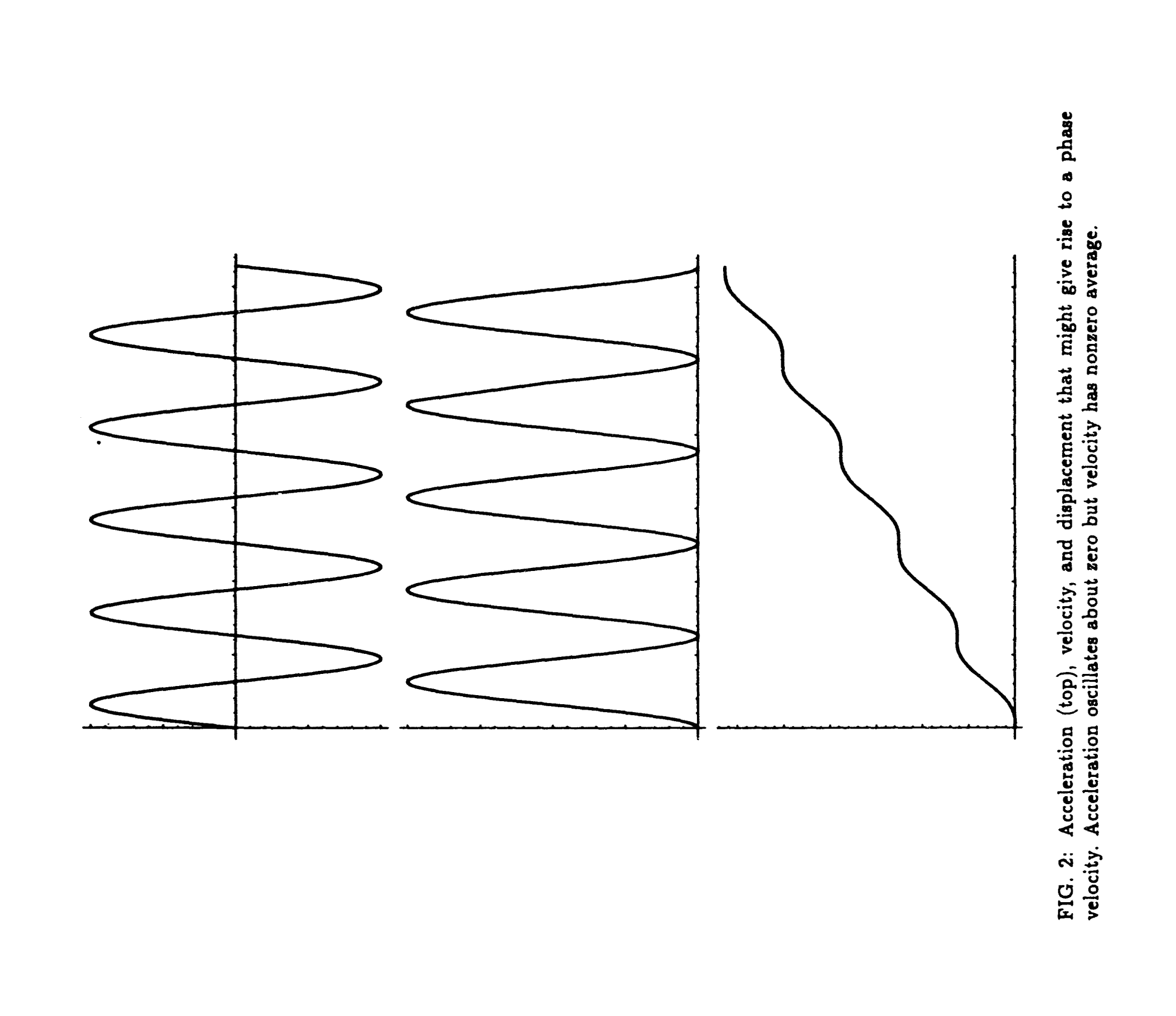


FIG. 3: "Cyclotron" motion of oscillating particles in uniform magnetic fields. In the first frame $a_{\text {applied }}=4.66 \times 10^{-4}(10 \mathrm{~T})$. In next frame $a_{\text {applied }}=0.466$; in last frame $a_{\text {applied }}=4.66$. The strangely shaped orbit in the last frame is only repeated a few times before the motion changes markedly. The figures are scaled approximately proportionally to the magnetic field strength. 


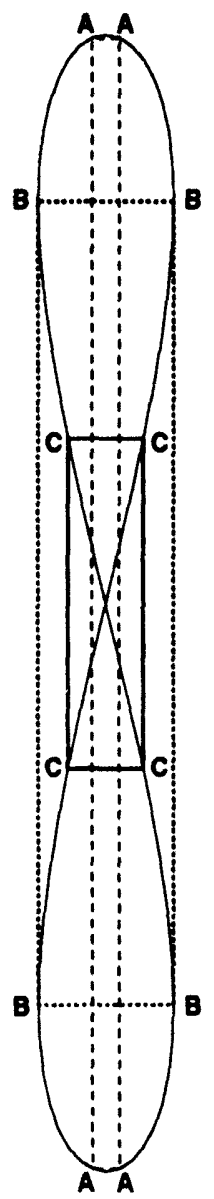

FIG. 4: Rectangles of points with similar dynamical variables. Three sample rectangles are drawn in the picture. 


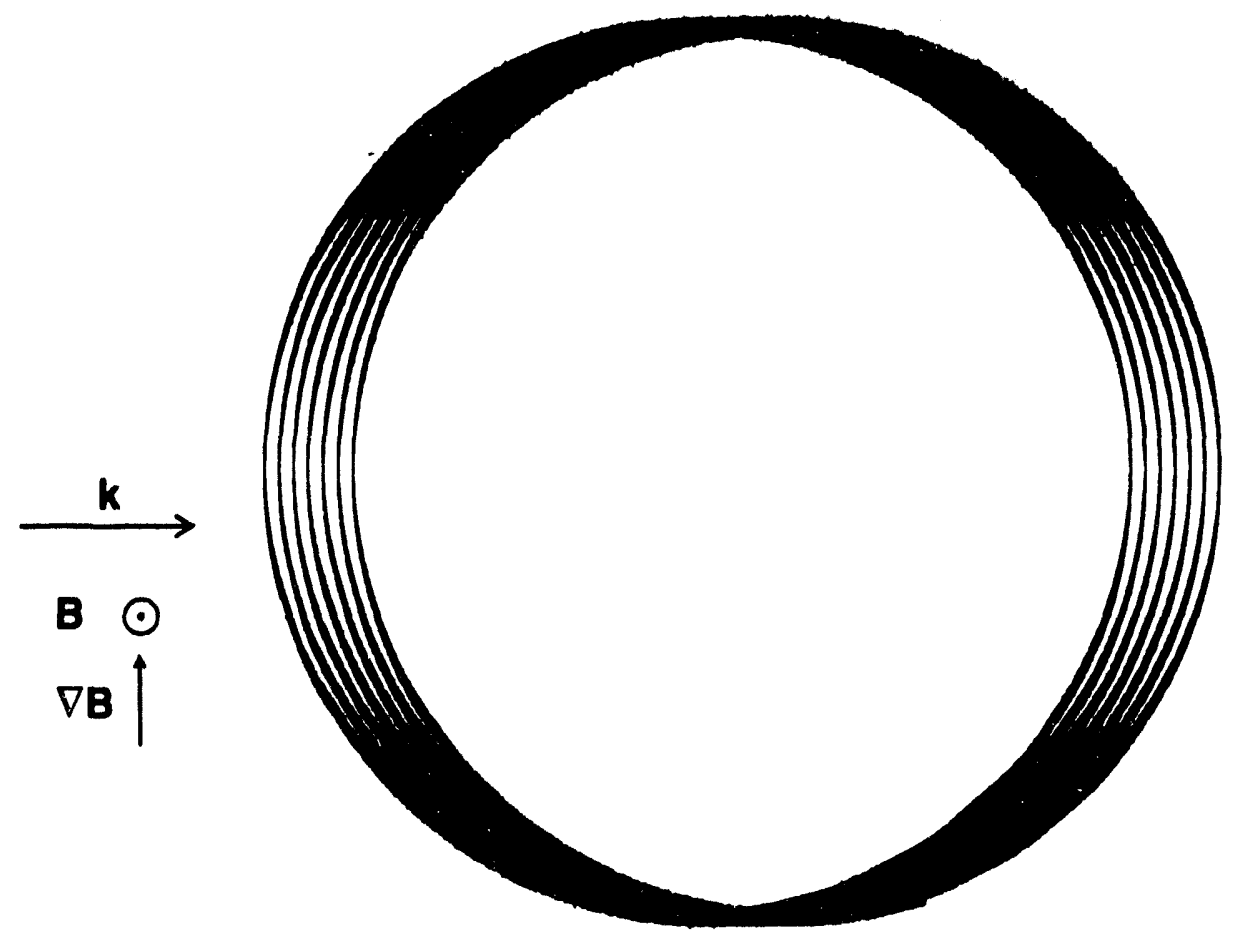

FIG. 5: Computer plot of gradient-B drift of oscillating electron in spatially varying magnetic field. The magnetic field varies linearly with scale length $100 \lambda$. 


\section{EXTERNAL DISTRIBUTION IN ADDITION TO UC-420}

Dr. F. Paoloni, Univ. of Wollongong, AUSTRALIA

Prof. M.H. Brennan, Univ. of Sydney, AUSTRALIA

Plasma Research Lab., Australian Nat. Univ., AUSTRALIA

Prot. I.R. Jones, Flinders Univ, AUSTRALIA

Prof. F. Cap, Inst. for Theoretical Physics, AUSTRIA

Prof. M. Heindler, Institut für Theoretische Physik, AUSTRIA

Prot. M. Goossens, Astronomisch InstituUt, BELGIUM

Ecole Royale Militaire, Lab. de Phy. Plasmas, BELGIUM

Commission-European, DG. XII-Fusion Prog., BELGIUM

Prof. R. Bouciqué, Rijksuniversiteit Gent, BELGIUM

Dr. P.H. Sakanaka, Instituto Fisica, BRAZIL

Prof. Dr. I.C. Nascimento, Instituto Fisica, Sao Paulo, BRAZIL Instituto Nacional De Pesquisas Espaciais-INPE, BRAZIL Documents Office. Atomic Energy of Canada Ltd., CANADA Ms. M. Morin, CCFMTokamak de Varennes, CANADA

Dr. M.P. Bachynski, MPB Technologies, Inc., CANADA

Dr. H.M. Skarsgard, Univ. of Saskatchewan, CANADA

Prof. J. Teichmann, Univ. of Montreal, CANADA

Prof. S.R. Sreenivasan, Univ. of Calgary, CANADA

Prof. T.W. Johnston, INRS-Energie, CANADA

Dr. R. Bolton, Centre canadien de lusion magnétique, CANADA

Dr. C.R. James, Univ. of Alberta, CANADA

Dr. P. Lukác, Komenského Universzita, CZECHO-SLOVAKIA

The Librarian, Culham Laboratory, ENGLAND

Library, R61, Ruthertord Appleton Laboratory, ENGLAND

Mrs. S.A. Hutchinson, JET Library, ENGLAND

Dr. S.C. Sharma, Univ. of South Pacific, FIJI ISLANDS

P. Mähönen, Univ. of Helsinki, FINLAND

Prof. M.N. Bussac, Ecole Polytechnique., FRANCE

C. Mouttet, Lab. de Physique des Milieux lonisés, FRANCE

J. Radet, CEN/CADARACHE - Bat 506, FRANCE

Prof. E. Economou, Univ. of Crete, GREECE

Ms. C. Rinni, Univ. of loannina, GREECE

Preprint Library, Hungarian Academy of Sa., HUNGARY

Dr. B. DasGupta, Saha Inst. of Nuclear Physics, INDIA

Dr. P. Kaw, Inst. for Plasma Research, INDIA

Dr. P. Rosenau, Israel Inst. of Technology, ISRAEL

Librarian, Intemational Center for Theo Physics, ITALY

Miss C. De Palo, Associazione EURATOM-ENEA, ITALY

Dr. G. Grosso, Istituto di Fisica del Plasma, ITALY

Prof. G. Rostangni, Istituto Gas Ionizzati Del Cnr, ITALY
Dr. H. Yamato, Toshiba Res \& Devel Center, JAPAN

Prof. I. Kawakami, Hiroshima Univ., JAPAN

Prof. K. Nishikawa, Hiroshima Univ., JAPAN

Librarian, Naka Fusion Research Establishment, JAERI, JAPAN

Director, Japan Atomic Energy Research Inst., JAPAN

Prof. S. Itoh, Kyushu Univ., JAPAN

Research Info. Cr., National Instit. for Fusion Science, JAPAN

Prof. S. Tanaka, Kyoto Univ., JAPAN

Library, Kyoto Univ., JAPAN

Prof. N. Inove, Univ. of Tokyo, JAPAN

Secretary, Plasma Section, Electrotechnical Lab., JAPAN

S. Mori, Technical Advisor, JAERI, JAPAN

Dr. O. Mitarai, Kumamoto Inst. of Technology, JAPAN

Dr. G.S. Lee, Korea Basic Sci. Ctr., KOREA

J. Hyeon Sook, Korea Alomic Energy Research Inst., KOREA

D.I. Choi, The Korea Adv. Inst. of Sai. \& Tech., KOREA

Prof. B.S. Liley, Univ. of Waikato, NEW ZEALAND Inst of Physics, Chinese Acad Sci PEOPLE'S REP. OF CHINA Library, Inst. of Plasma Physics, PEOPLE'S REP. OF CHINA Tsinghua Univ. Library, PEOPLE'S REPUBLIC OF CHINA

Z. Li, S.W. Inst Physics, PEOPLE'S REPUBLIC OF CHINA

Prol. J.A.C. Cabral, Instituto Superior Tocnico, PORTUGAL

Prot. M.A. Hellberg, Univ. of Natal, S. AFRICA

Prot. D.E. Kim, Pohang Inst. of Sci. \& Tech., SO. KOREA

Prot. C.I.E.M.A.T, Fusion Division Library, SPAIN

Dr. L. Stenflo, Univ. of UMEA, SWEDEN

Library, Royal Inst. of Technology, SWEDEN

Prof. H. Wilhelmson, Chalmers Univ. of Tech., SWEDEN

Centre Phys. Des Plasmas, Ecole Polytech, SWITZERLAND

Bibliotheek, inst. Voor Plasma-Fysica, THE NETHERLANDS

Asst. Prof. Dr. S. Cakir, Middle East Tech. Univ., TURKEY

Dr. V.A. Glukhikh,Sei. Res. Inst. Electrophys.I Apparatus, USSR

Dr. D.D. Ryutov, Siberian Branch of Academy of Sci., USSR

Dr. G.A. Eliseev, I.V. Kurchatov Inst., USSR

Librarian, The Ukr.SSR Academy of Sciences, USSR

Dr. L.M. Kovrizhnykh, Inst. of General Physics, USSR

Kemforschungsanlage GmbH, Zentralbibliothek, W. GERMANY

Bibliothek, lisst. Für Plasmaforschung, W. GERMANY

Prot. K. Schindler, Ruhr-Universitát Bochum, W. GERMANY

Dr. F. Wagner, (ASDEX), Max-Planck-Institut, W. GERMANY

Librarian, Max-Planck-Institut, W. GERMANY 

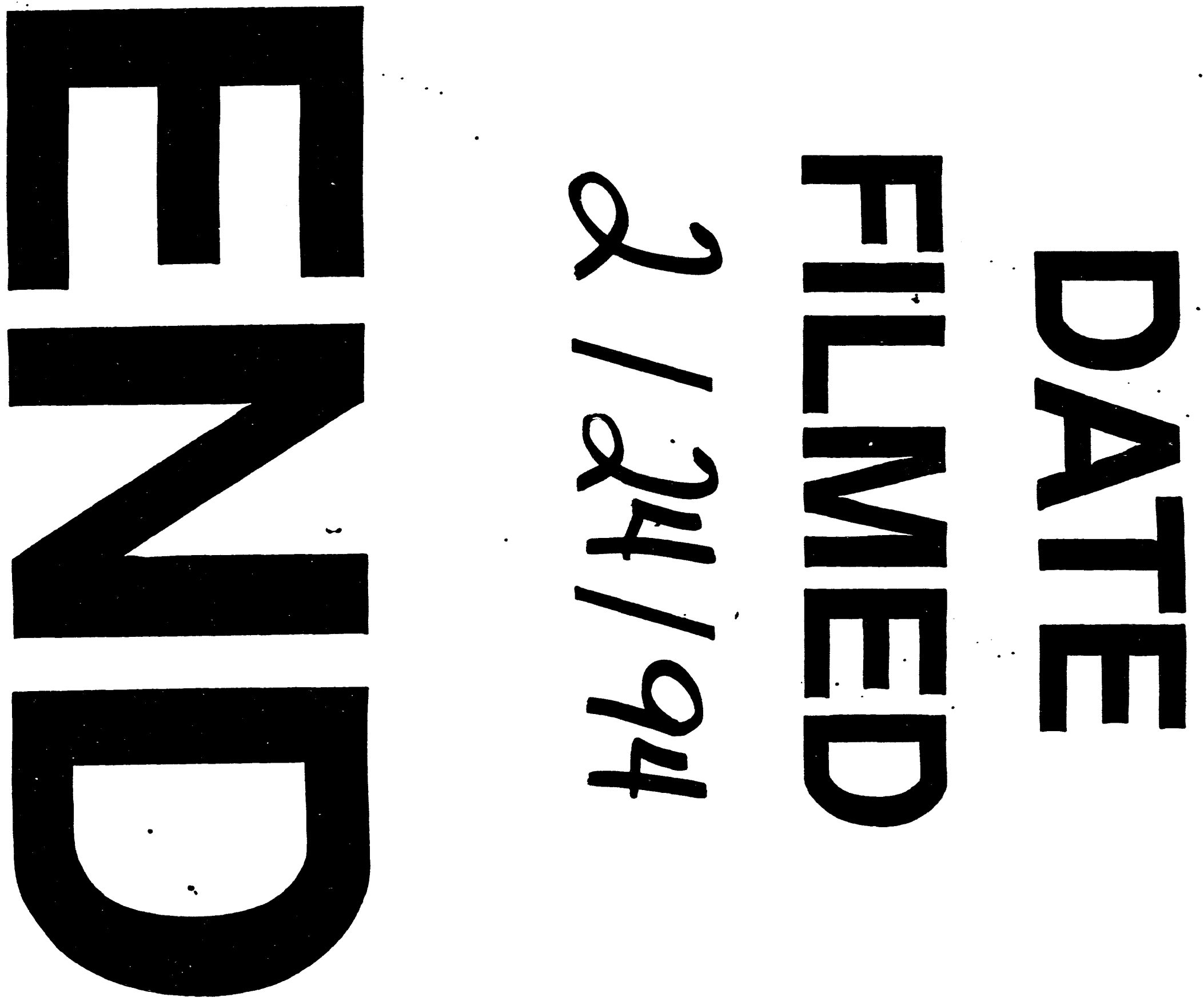
10

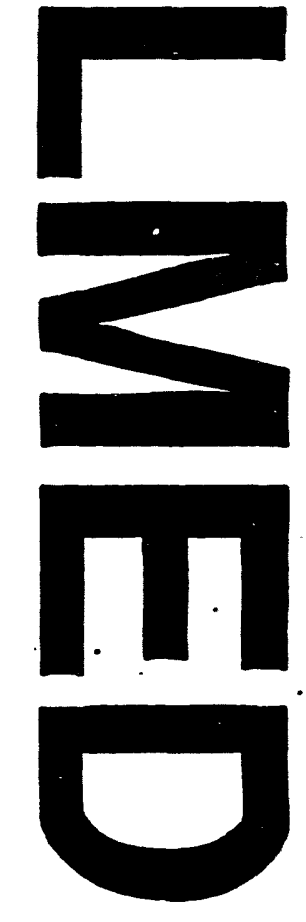




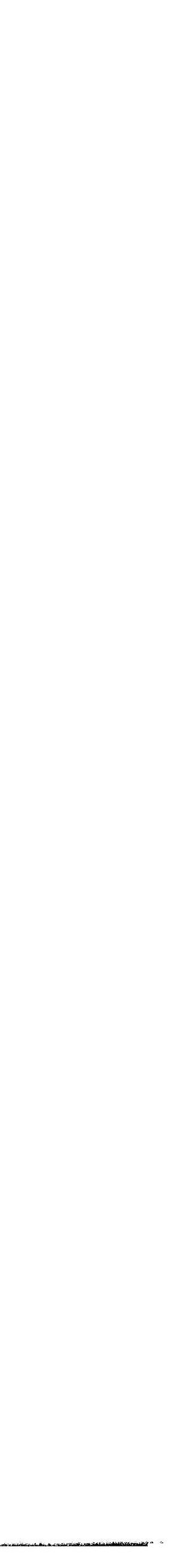

